\title{
Hyphal Wall Growth in Neurospora crassa and Geotrichum candidum
}

\author{
By A. P. J. TRINCI AND ANNETTE J. COLLINGE \\ Microbiology Department, Queen Elizabeth College, University of London, London W8 7 AH
}

(Received I5 April 1975; revised 16 June 1975)

\begin{abstract}
SUMMARY
Growth of the walls of hyphae of Neurospora crassa and Geotrichum candidum was studied using longitudinal and serial transverse sectioning methods. Rigidification of the hyphal wall below the extension zone did not appear to involve the gross formation of a secondary wall since the transition from extensible to non-extensible wall was not associated with an increase in thickness. However, behind the extension zone the walls of leading hyphae of $N$. crassa increased in thickness until eventually they attained a thickness which was up to five times that of the tip wall. A hypothesis of hyphal wall growth is proposed.
\end{abstract}

\section{INTRODUCTION}

Hyphal growth is polarized, i.e. extension is confined to the hyphal tip (Trinci \& Banbury, 1967). The length of the apical extension zone of a hypha may be determined by measuring the length of the tapered region of its tip (Trinci \& Halford, 1975). The tips of fungal hyphae contain vesicles (Grove $\&$ Bracker, 1970) which are assumed to carry wall precursors and/or the enzymes required for the insertion of these precursors into the existing wall (BartnickiGarcia, 1973). The vesicles are also thought to supply the membrane which enables the plasmalemma at the tip to expand rapidly in surface area. It has been estimated that about 38000 vesicles fuse with a tip of a Neurospora crassa hypha (wild-type) during I min of extension growth at $25^{\circ} \mathrm{C}$ (Collinge \& Trinci, I974). The vesicles and other precursors required to maintain the high apical extension rate observed in fungi are probably generated throughout the peripheral growth zone (Trinci, I97I) of the hypha (Collinge \& Trinci, 1974). It is not known if increase in wall thickness behind the tip (i.e. secondary wall growth) involves this vesicular system (Collinge \& Trinci, 1974).

Robertson (1959) has proposed that hyphal extension involves a balance between the insertion of new wall material at the hyphal tip and rigidification of the newly formed wall at the base of the extension zone. The rigidification process may involve the addition of nonextensible, secondary wall material to the extensible primary wall and/or the formation of cross-linkage between the existing wall polymers of the primary walls. Robertson (I968) favours the hypothesis that deposition of secondary wall material is involved in the rigidification process.

This study was undertaken to determine if there was an increase in wall thickness at the hyphal tip in the region of transition from extensible to non-extensible wall. Studies were made on hyphae of undifferentiated mycelia (mycelia formed during the early exponential stage of growth on solid medium; Steele \& Trinci, 1975) and the 'leading' hyphae at the margin of 'mature' colonies. 


\section{METHODS}

Organisms and media. The spreading colonial mutants of Neurospora crassa (spco-I and -12) and the colonial temperature-sensitive mutant (cot-3) were supplied by the Fungal Genetics Stock Center, Humboldt State College Foundation, Arcata, California, U.S.A.; cot-3 has wild-type characteristics at $25{ }^{\circ} \mathrm{C}$. The wild-type strain of $N$. crassa (SYR-I7-3A) (inos $+C, D, E$ ) was kindly supplied by Professor E. L. Tatum. Geotrichum candidum (Queen Elizabeth College strain FI, formerly called G. lactis) was also used in this investigation. The $N$. crassa strains were grown on Vogel's minimal medium (Vogel, 1956$)$ with $\mathrm{I} \%(\mathrm{w} / \mathrm{v})$ sucrose as the carbon source; this medium was prepared as described previously (Trinci, 1973). Geotrichum candidum was grown on DM medium (Trinci, 197I).

Undifferentiated hyphae (Steele \& Trinci, 1975) of $N$. crassa cot-3 and G. candidum were grown on the surface of solid media at $25{ }^{\circ} \mathrm{C}$; the hyphae were fixed in $4 \%(\mathrm{v} / \mathrm{v})$ glutaraldehyde at $25^{\circ} \mathrm{C}$ (Trinci \& Collinge, 1973) at 27 and $16 \mathrm{~h}$ after inoculation respectively. The total hyphal length of the mycelia did not exceed more than about $5 \mathrm{~mm}$. 'Mature' colonies were grown at $25^{\circ} \mathrm{C}$ on solid media and the 'leading' hyphae at the margin of the colony were fixed whilst in the linear phase of growth. Procedures employed for electron microscopy have been described previously (Trinci \& Collinge, I973; Collinge \& Trinci, 1974). Measurements of wall thickness were made upon photographs of transverse hyphal sections; the hyphae had a more-or-less circular outline in these sections. The sections were usually silver or gold in colour and thus varied in thickness from about 60 to about $150 \mathrm{~nm}$. The values for the thickness of the wall at various distances from the hyphal tip (Figs. I, 6 and 7) are always the means of five measurements made around the hyphal circumference.

\section{RESULTS}

Wall thickness of 'leading' hyphae at the margin of 'mature' colonies of Neurospora crassa

Figures I, 3 and 4 show the wall thickness and diameter of 'leading' hyphae at the margin of a 'mature' colony of $N$. crassa cot-3. The hypha in Fig. I had an extension zone of about $28 \mu \mathrm{m}$ but there was no appreciable change in wall thickness over the apical $56 \mu \mathrm{m}$ of the hypha. The wall thickness of 'leading' hyphae of the wild-type $N$. crassa (SYR-I 7-3A) also did not vary over the terminal $50 \mu \mathrm{m}$ of the hypha. In longitudinal sections the wall over the apical dome of the hypha was sometimes thicker than the wall of the basal part of the extension zone. The 'leading' hyphae of wild-type and spreading colonial mutants (spco) of $N$. crassa had tip walls of about the same thickness (Table I).

The walls of 'leading' hyphae of $N$. crassa cot-3 showed an appreciable increase in thickness about $500 \mu \mathrm{m}$ from the margin of the colony (Fig. I) and finally attained a thickness (about $270 \mathrm{~nm}$ ) which was more than five times that of the tip wall.

\section{Wall thickness of hyphae of undifferentiated mycelia of Neurospora crassa cot-3 and Geotrichum candidum}

Figures 2 and 6 show the change in wall thickness and diameter of hyphae of undifferentiated mycelia of $N$. crassa cot-3 as observed in serial transverse sections (Fig. $6 b$ ) and a median longitudinal section (Fig. 6a). These hyphae were narrower and had shorter extension zones (about $2 \mu \mathrm{m}$ ) than the 'leading' hyphae at the margin of 'mature' colonies (about $28 \mu \mathrm{m}$ ). There was no significant change in wall thickness over the terminal $10 \mu \mathrm{m}$ of the hyphae but between about 10 to about $200 \mu \mathrm{m}$ from the tip there was a gradual increase in thickness until the wall attained a more-or-less constant thickness of 120 to $140 \mathrm{~nm}$. The 


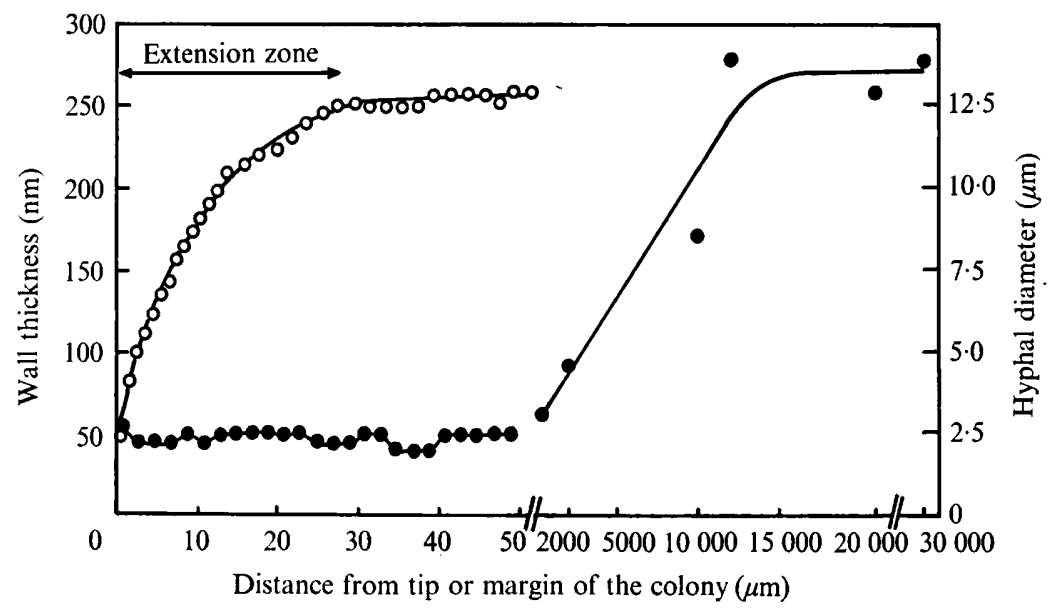

Fig. I. Wall thickness $(\bullet)$ and diameter $(\bigcirc)$ of ' leading' hyphae at the margin of 'mature' colonies of $N$. crassa cot-3 at $25^{\circ} \mathrm{C}$. Measurements from o to $50 \mu \mathrm{m}$ were made on a median longitudinal section of a single hypha. Measurements from $500 \mu \mathrm{m}$ to $30 \mathrm{~mm}$ from the margin of the colony were made on different 'leading' hyphae.

Table I. Thickness of the tip walls of 'leading' hyphae of N. crassa at the margin of 'mature' colonies and hyphae of undifferentiated mycelia

\begin{tabular}{llcc}
\multicolumn{1}{c}{ Strain } & Type of hypha & O-5 $\mu \mathrm{m}$ from tip & 5-10 $\mu \mathrm{m}$ from tip \\
SYR-1 7-3A (wild-type) & 'Leading', & 45 & $5 \mathrm{I}$ \\
spco-I & 'Leading', & 50 & 46 \\
spco -12 & 'Leading', & 49 & 56 \\
cot-3 & 'Leading' & 50 & 46 \\
cot-3 & Undifferentiated & 65 & 63 \\
& $*$ Mean of at least 15 measurements.
\end{tabular}

* Mean of at least 15 measurements.

tip walls of immature hyphae of $N$. crassa were slightly thicker than those of the 'leading' hyphae of the same strain (Table I). In regions distal from the tip, the walls of hyphae of undifferentiated mycelia of $N$. crassa were made up of an electron-dense inner layer and an electron-transparent outer layer (Fig. $2 d$ ); 200 to $1500 \mu \mathrm{m}$ from the tip, where the wall thickness was approximately constant (Fig. 6), the inner layer had a mean thickness of $5 \mathrm{I} \pm \mathrm{IO} \mathrm{nm}$ and the outer layer of $87 \pm \mathrm{I} 2 \mathrm{~nm}$. The vesicles at the tip of immature hyphae of $N$. crassa had a mean diameter of $62 \pm \mathrm{I} 3 \mathrm{~nm}$ and were thus smaller than the vesicles (mean diameter $134 \pm 26 \mathrm{~nm}$ ) at the tips of the 'leading' hyphae of this same strain (Collinge \& Trinci, 1974).

Figures 5 and 7 show the wall thickness, diameter and vesicle concentration of hyphae of an undifferentiated mycelium of $G$. candidum. Like $N$. crassa, these hyphae had extension zones of about $2 \mu \mathrm{m}$. The concentration of vesicles in the cytoplasm only increased in the regions where the diameters of the hyphae narrowed, i.e. in the extension zones. The vesicles of $G$. candidum had a mean diameter of $76 \pm 20 \mathrm{~nm}$. Longitudinal sections showed that the vesicles were not elongated in the direction of the long axis of the hypha.

The thickness of the hyphal wall of G. candidum remained constant (about $70 \mathrm{~nm}$ ) over the terminal $300 \mu \mathrm{m}$ of the hypha (Figs. 5 and 7 ). Other sections showed that the wall of this particular hypha had not increased in thickness at distances of up to $\mathrm{I} \cdot 4 \mu \mathrm{m}$ from the tip. The 

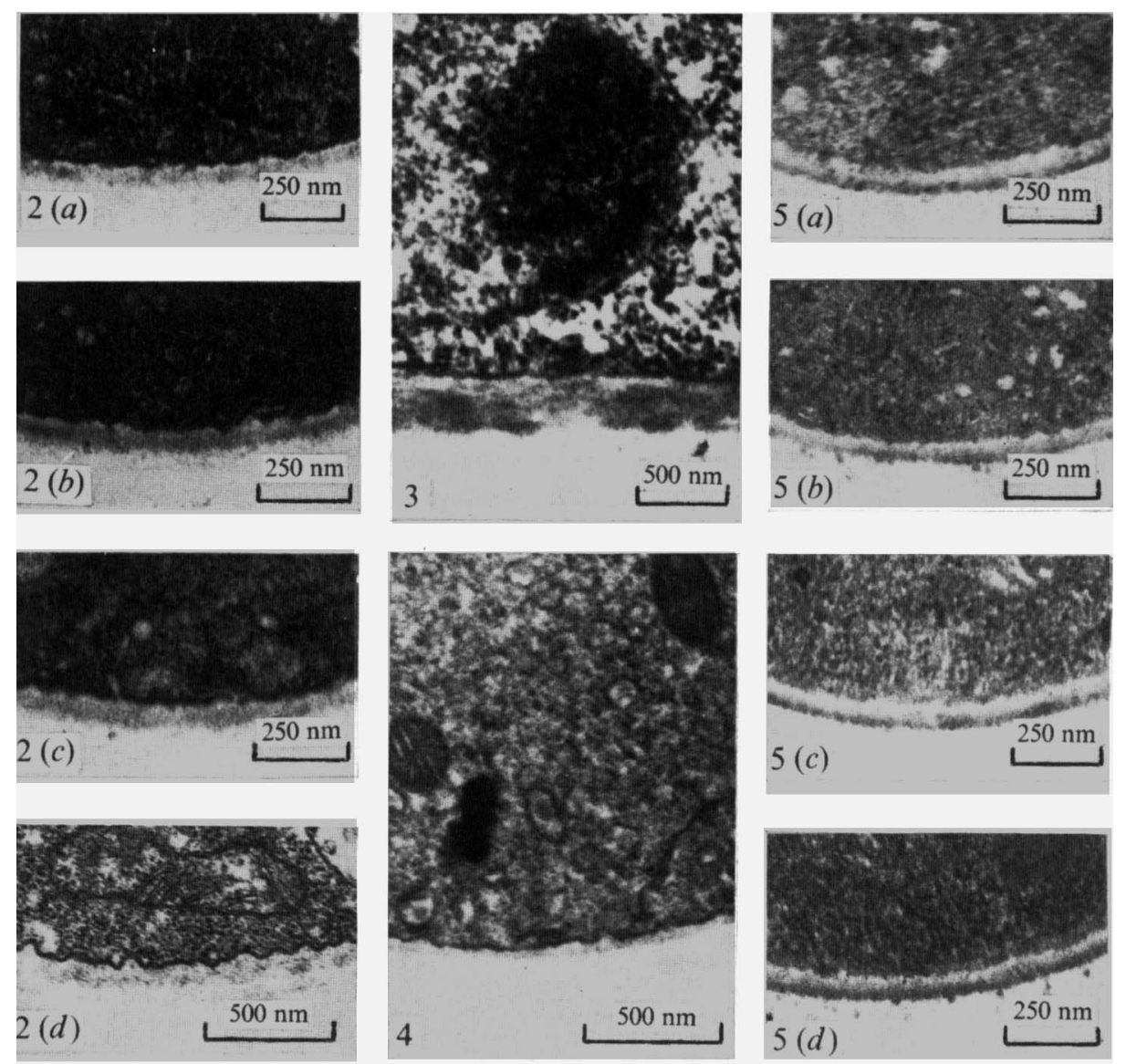

Fig. 2. Transverse sections of the wall of an undifferentiated hypha of $N$. crassa cot -3 at various distances (about $2 \cdot 5,4 \cdot 0,5^{\circ} 0$ and $750 \mu \mathrm{m}$ in $a, b, c$ and $d$, respectively) from the tip.

Fig. 3. Longitudinal section of the wall of a 'leading' hypha (this hypha had a diameter of II $\mu \mathrm{m}$ ) of $N$. crassa about $20 \mathrm{~mm}$ from the margin of the colony.

Fig. 4. Transverse section of the wall of a 'leading' hypha of N. crassa cot-3 about $3 \mu \mathrm{m}$ from the tip.

Fig. 5. Transverse sections of the wall of an undifferentiated hypha of $G$. candidum at various distances (about $\mathrm{I}, \mathrm{I} 0,30$ and $\mathrm{I} 50 \mu \mathrm{m}$ in $a, b, c$ and $d$, respectively) from the tip.

apparent increase in wall thickness (Fig. 7) over the hyphal dome (the apical I $\mu \mathrm{m}$ ) can almost certainly be accounted for in terms of the curvature of the wall at the tip. Throughout the terminal $300 \mu \mathrm{m}$ of the hypha the wall was made up of two layers (Fig. 5) of more or less equal thickness: an electron-transparent inner layer and an electron-dense outer layer.

\section{DISCUSSION}

The results obtained with $N$. crassa and $G$. candidum suggest that rigidification of the wall below the extension zone does not involve the formation of a substantial secondary wall. Published electron micrographs of longitudinal sections of hyphae of other species also indicate that there is no appreciable change in wall thickness in areas at the base and immediately below the extension zone (Grove \& Bracker, 1970). The apparent increase in wall 


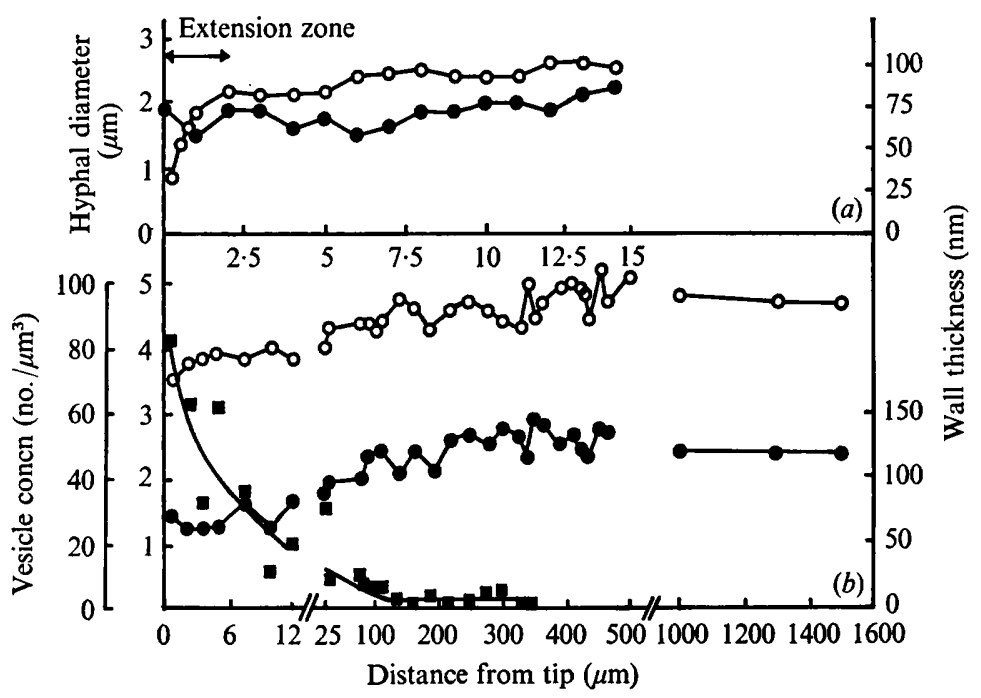

Fig. 6. Wall thickness $(\mathcal{O})$, diameter $(\bigcirc)$ and vesicle concentration $(\boldsymbol{\square})$ of undifferentiated hyphae ( 27 h culture) of $N$. crassa cot -3 at $25^{\circ} \mathrm{C}$. (a) Measurements made on a median longitudinal section of a single hypha; $(b)$ measurements made on serial transverse sections of a single hypha.

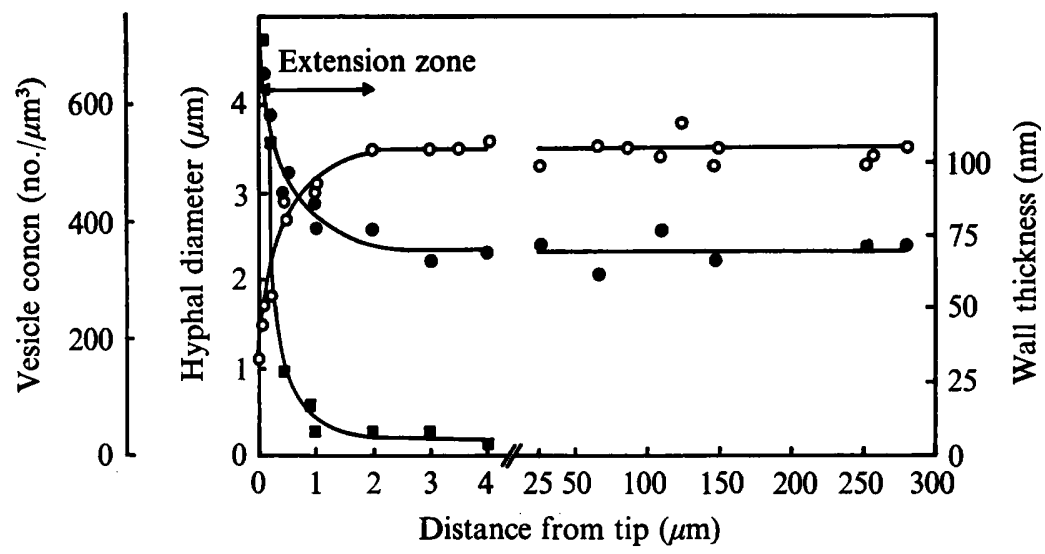

Fig. 7. Wall thickness (O), diameter $(\bigcirc)$ and vesicle concentration $(\square)$ of an undifferentiated ( $16 \mathrm{~h}$ culture) hypha of $G$. candidum. Measurements made on serial transverse sections of a single hypha.

thickness which was sometimes observed over the apical domes of hyphae cut longitudinally was probably caused by the non-median nature of these particular sections (see McClure, Park \& Robinson, 1968). Rigidification of the wall below the extension zone may involve the addition of small amounts of substances to the wall which do not increase its thickness appreciably, and/or the formation of cross-linkages between existing wall polymers. It is possible that the primary wall only remains extensible whilst vesicles (containing lytic enzymes?) continue to fuse with it. Rigidification may thus occur at the base of the extension zone simply because vesicles are no longer fusing with the wall at this point.

In N. crassa (Figs. I and 6), but not apparently in G. candidum (Fig. 7), there is an increase in wall thickness behind the hyphal tip due to secondary wall growth. D. Hunsley (personal communication) also observed changes in wall thickness in $N$. crassa similar to those we 


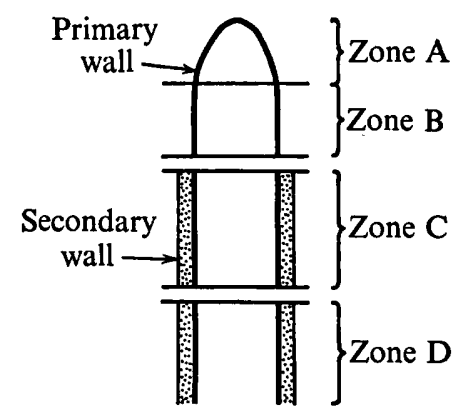

Fig. 8. Tentative model of hyphal wall growth based mainly upon studies with $N$. crassa. Zone A: tapered extension zone with a primary wall, containing microfibrils, which becomes progressively less extensible with distance from the hyphal apex. Wall thickness maintained approximately constant and extensible by the fusion with it of vesicles containing wall precursors and/or lytic and synthetic enzymes. Hyphal extension dependent upon the rate of supply of vesicles. Zone B: region of constant hyphal diameter. The wall is inextensible and of approximately the same thickness as in zone A. Rigidification of the wall may involve the formation of cross-linkages between existing wall polymers or the addition of small quantities of new wall material. It is possible that vesicles do not normally fuse with this region of the hyphal wall. Zone $\mathrm{C}$ : region of secondary wall formation. The wall increases in thickness with distance from the tip, eventually becoming a 'mature' wall of approximately constant thickness. Zone D: under conditions of carbon starvation (Zonneveld, 1972) some of the wall polymers may be degraded to provide substrates for endogenous metabolism.

report. The observation that the inner layer of the 'mature' hyphal wall of $N$. crassa (present results, and Hunsley \& Burnett, 1970) has approximately the same thickness (about $50 \mathrm{~nm}$ ) as the primary tip wall (Table I) suggests that the former may be continuous with the latter (Fig. 8).

The chitin content of the inner layer of the 'mature' hyphal wall of $N$. crassa (Hunsley \& Burnett, 1970) provides further evidence in support of the above hypothesis, since the tips of hyphae of $N$. crassa and other fungi are covered by a microfibrillar mesh of chitin (Hunsley \& Burnett, 1970). The chemistry of the secondary wall probably differs both qualitatively and quantitatively from that of the primary wall (Hunsley \& Burnett, 1970). Support for these contentions and the hypothesis presented in Fig. 8 is provided by the observations that walls at the tips of fungal hyphae differ from those below the tip both antigenically (Fultz \& Sussman, 1966) and in their reaction to the fluorescent brightener, Calcofluor (Gull \& Trinci, 1974). The glycoprotein reticulum of the walls of hyphae of $N$. crassa appears to develop progressively behind the tip as a superficial wall layer (Hunsley \& Burnett, 1970).

Thus our results and those of Hunsley \& Burnett (1970) and Hunsley (1973) suggest that hyphal walls of $N$. crassa and Phytophthora parasitica increase in thickness largely or wholly by secondary wall layers being deposited upon the existing primary wall (Fig. 8). The possibility that secondary wall material may sometimes (e.g. during unbalanced growth and differentiation processes such as spore formation) be deposited on the cytoplasmic side of the primary wall is not excluded.

As observed by Robertson (I965), 'The key to the fungal hypha lies in the apex.' Certainly, the crucial events involved in hyphal morphogenesis probably occur in the apical and immediate sub-apical areas rather than in regions of secondary wall formation far removed from the tip. Thus it is unlikely that comparison of the gross chemistry of 'mature' hyphal walls (e.g. De Terra \& Tatum, I963) will reveal the subtle chemical changes at the hyphal tip which are responsible for a particular type of morphogenetic change. 
We thank Dr D. Hunsley for helpful discussion, and the Royal Society and the Science Research Council for financial support.

\section{REFERENCES}

BARTNICKI-GARCIA, S. (I973). Fundamental aspects of hyphal morphogenesis. In Microbial Differentiation. Edited by J. M. Ashworth and J. E. Smith. Cambridge: Cambridge University Press.

ColuINGe, A. J. \& TRINCI, A. P. J. (1974). Hyphal tips of wild-type and spreading colonial mutants of Neurospora crassa. Archives of Microbiology 99, 353-368.

De Terra, N. \& TATUM, E. L. (I963). A relationship between cell wall structure and colonial growth in Neurospora crassa. American Journal of Botany 5o, 669-677.

Fultz, S. A. \& Sussman, A. S. (1966). Antigenic differences in the surface of hyphae and rhizoids in Allomyces. Science, New York 152, 785-787.

Grove, S. N. \& BRACKER, C. E. (1970). Protoplasmic organization of hyphal tips among fungi: vesicles and Spitzenkörper. Journal of Bacteriology 104, 989-1009.

Gull, K. \& TrINCI, A. P. J. (1974). Detection of areas of wall differentiation in fungi using fluorescent staining. Archives of Microbiology 96, 53-57.

HunsLey, D. (1973). Apical wall structure in hyphae of Phytophthora parasitica. New Phytologist 72, 985-990.

HunSLEY, D. \& BURNETr, J. H. (1970). The ultrastructural architecture of the walls of some hyphal fungi. Journal of General Microbiology 62, 203-218.

McCluRe, W. K., PARK, D. \& Robinson, P. M. (1968). Apical organization in the somatic hyphae of fungi. Journal of General Microbiology 50, $177-182$.

ROBERTSON, N. F. (1959). Experimental control of hyphal branching and branch form in Hyphomycetous fungi. Journal of the Linnaean Society (Botany) 56, 207-2 I I.

Robertson, N. F. (1965). The fungal hypha. Transactions of the British Mycological Society 48, I-8.

Robertson, N. F. (1968). The growth process in fungi. Annual Review of Phytopathology 6, I I 5-I 36.

Steele, G. C. \& TrincI, A. P. J. (1975). Morphology and growth kinetics of hyphae of differentiated and undifferentiated mycelia of Neurospora crassa. Journal of General Microbiology.

TRINCI, A. P. J. (197I). Influence of the width of the peripheral growth zone on the radial growth rate of fungal colonies on solid media. Journal of General Microbiology 67, 325-344.

TRINCI, A. P. J. (1973). Growth of wild-type and spreading colonial mutants of Neurospora crassa in batch culture and on agar medium. Archiv für Mikrobiologie 91, 1 I3-1 26.

TrincI, A. P. J. (1974). A study of the kinetics of hyphal extension and branch initiation of fungal mycelia. Journal of General Microbiology 81, 225-236.

Trincr, A. P. J. \& Banbury, G. A. (I967). A study of the growth of tall conidiophores of Aspergillus giganteus. Transactions of the British Mycological Society 50, 525-538.

TrincI, A. P. J. \& Collinge, A. J. (I973). Structure and plugging of septa of wild-type and spreading colonial mutants of Neurospora crassa. Archiv für Mikrobiologie 91, 355-364.

Trinci, A. P. J. \& Halford, E. A. (1975). The extension zone of stage I sporangiophores of Phycomyces blakesleeanus. New Phytologist 74, 8I-83.

Vogel, H. J. (1956). A convenient medium for Neurospora (medium N). Microbial Genetics Bulletin r3, $42-44$.

ZONNEVELD, B. J. M. (1972). Morphogenesis in Aspergillus nidulans: the significance of $\alpha-\mathrm{I}, 3$ glucan and $\alpha-\mathrm{I}, 3$ glucanase for cleistothecium development. Biochimica et biophysica acta 273, 174-187. 